\title{
Generation of induced pluripotent stem cells from renal tubular cells of a patient with Alport syndrome
}

This article was published in the following Dove Press journal: International Journal of Nephrology and Renovascular Disease 2I August 2015

Number of times this article has been viewed

\author{
Wenbiao Chen ${ }^{1, *}$ \\ Jianrong Huang $2, *$ \\ Xiangqi Yu' \\ Xiaocong $\operatorname{Lin}^{3}$ \\ Yong Dai'
}

'The Clinical Medical Research Center, Second Clinical Medical College of Jinan University, Shenzhen People's Hospital, ${ }^{2}$ Department of Hemodialysis, The Third People's Hospital of Shenzhen, Shenzhen, Guangdong, ${ }^{3}$ Institute of Biochemistry and Molecular Biology, Guangdong Medical College, Zhanjiang, People's Republic of China

*These authors contributed equally to this work

Correspondence: Yong Dai

The Clinical Medical Research Center, Second Clinical Medical College of Jinan University, Shenzhen People's Hospital, Shenzhen 518020, People's Republic of China

Email daiyong2222@gmail.com

\begin{abstract}
Alport syndrome (AS) is a hereditary disease that leads to kidney failure and is caused by mutations in the COL4A3,COL4A4, and COL4A5 genes that lead to the absence of collagen $\alpha 3 \alpha 4 \alpha 5$ (IV) networks in the mature kidney glomerular basement membrane. Approximately $80 \%$ of AS is X-linked because of mutations in COL4A5, the gene encoding the alpha 5 chain of type IV collagen. To investigate the pathogenesis of AS at the genetic level, we generated induced pluripotent stem cells (iPSCs) from renal tubular cells of a patient with AS. The successful iPSC generation laid the foundation to master the repair of the COL4A5 gene and to evaluate the differentiation of iPSC into Sertoli cells and the accompanying epigenetic changes at each stage. The generation of iPSCs from AS patients not only confirms that iPSCs could be generated from renal tubular cells, but also provides a novel type of genetic therapy for AS patients. In this study, we generated iPSCs from renal tubular cells via ectopic expression of four transcription factors (Oct4, Sox2, c-myc, and Klf4). According to the human embryonic stem cell (hESC) charter, iPSC formation was confirmed by comparatively analyzing hESC markers via colony morphology, immunohistochemistry, qRT-PCR, flow cytometry, gene expression profiling of the three germ layers, and karyotyping. Our results demonstrated that iPSCs were similar to hESCs with regard to morphology, proliferation, hESC-specific surface marker expression, and differentiation into the cell types of the three germ layers. The efficient generation of iPSCs from the renal tubular cells of an AS patient would provide a novel model to investigate the mechanisms underlying AS and to develop new treatments for AS.
\end{abstract}

Keywords: Alport syndrome, induced pluripotent stem cells, renal tubular cells

\section{Introduction}

Alport syndrome (AS) is an inherited disease that results in kidney failure, hearing loss, and ocular abnormality. ${ }^{1} \mathrm{AS}$ is caused by mutations of the genes encoding the key collagen chains $\alpha 3, \alpha 4, \alpha 5$, which are necessary for the composition of collagen type IV to form the kidney glomerular basement membrane. ${ }^{2}$ Glomerular basement membrane contains an $\alpha 3, \alpha 4, \alpha 5$, network that is essential for the maintenance of kidney ultrafiltration function. The absence of this network in patients with AS leads to progressive glomerulonephritis. ${ }^{3}$ It has been reported that AS is the most common genetic disease in the kidney and that it inevitably leads to end-stage renal disease with a subsequent need for kidney transplantation and dialysis therapy. ${ }^{4}$ The diagnosis of AS relies on history, physical examination, immunohistochemical analysis, and histologic studies. However, the diagnosis of AS still relies heavily on histological analysis of renal biopsy samples by using electron microscopy. ${ }^{5}$ Because AS is heterogeneous at the clinical and genetic levels, molecular genetics could serve as a powerful tool for 
a definite diagnostic workup in patients. ${ }^{6}$ Novel therapeutic approaches may eventually provide a treatment or cure for these patients, thereby avoiding the need for transplantation and dialysis.

In this study, we successfully generated induced pluripotent stem cells (iPSCs) from the renal tubular cells of AS patients, which provided an experimental basis to further research the pathogenesis of AS at the genetic level. We used the iPSCs to repair the mutated gene, and we induced the iPSCs to differentiate into Sertoli cells. The iPSCs may thus help to identify the factors important for the regulation of cell differentiation and to develop novel genetic treatments for AS. The generation of iPSCs from systemic lupus erythematosus patients has been reported, ${ }^{7}$ and the generation of iPSCs from fibroblasts has also been successfully achieved. ${ }^{8}$ Our results not only demonstrate that iPSCs could be generated from cultured human cells with the same characteristics as human embryonic stem cells (hESCs), but also provide a potential method to further research the pathogenesis, diagnosis, and treatment of AS.

\section{Material and methods AS family analysis}

In our previous research, ${ }^{9}$ we identified an AS family that contained three patients and five healthy individuals. The proposita (III2) was female and aged 26 years and was diagnosed with AS at Second Clinical College of Jinan University in 2013. Clinical examination revealed gross hematuria and albuminuria. The pathological examination under light microscopy and electron microscopy confirmed that the nephron of III2 presented pathological changes indicative of AS (Figure 1). The proposita's grandmother (I2) also suffered from AS and had died of kidney failure. The proposita's mother (II3) had evident AS clinical symptoms, including kidney failure, gross hematuria and albuminuria, sensorineural hearing loss, and pathognomonic ocular lesions. The proposita's sister (III3) also presented mild gross hematuria and albuminuria (Figure 2). The results of the routine blood examination, urine routine test and blood biochemistry as well as the general information for all family members are listed in Table 1. To determine whether mutation of the COL4A5 gene was the reason for familial AS, DNA sequence analysis was performed for the entire coding region and exon-intron boundaries of the COL4A5 gene. The sequence analysis identified a novel splicing mutation of c. 1517-I G>T in the COL4A5 gene causing AS (Figure 3). In our study, we selected three AS patients (III2, III3, and III) and three healthy individuals (III1, III4, and II4) for evaluation. We used the proposita (III2) as a representative example for generation and characterization of iPSCs. This study was approved by the guidelines of Jinan University, which were in accordance with the Helsinki Declaration for Ethical Principles for Medical Research Involving Human Subjects. The participants also provided informed consent.

\section{Urine collection and cell culture}

Before urine collection, the proposita was asked to wash her vulva with soapy water. The external vaginal and urethral openings were subjected to regular disinfection. Further, a urinary catheter was surgically inserted. All operations were performed under a sterile environment. We aseptically collected $200 \mathrm{~mL}$ of midstream urine during micturition
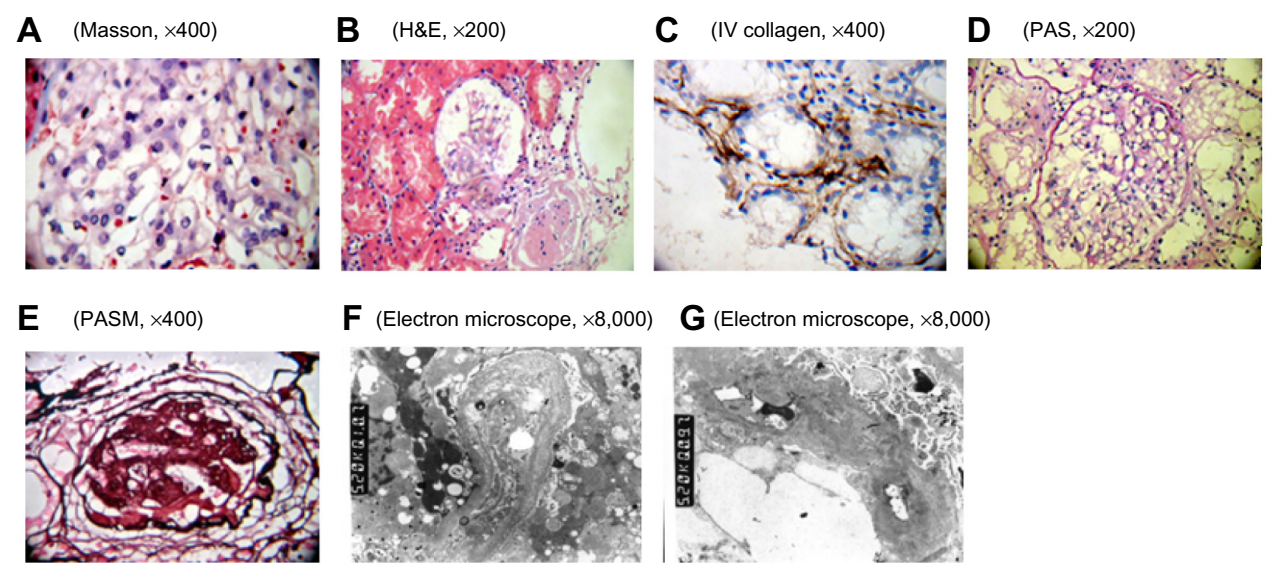

Figure I Pathological examination of the kidney by using light microscopy and electron microscopy.

Notes: (A) mesangial mild proliferation; (B) mesangial mild proliferation and glomerulosclerosis; (C) renal tubular atrophy and renal tubular basement membrane type IV collagen deposition; (D) GBM thickening; (E) glomerular sclerosis; (F and $\mathbf{G})$ irregular thickening, thinning, and splitting of GBM. Electron-dense material deposited in the membrane. Mesangial matrix severely increased. There were dense deposits showing cord-like distribution. Part of tubular cell vacuolar degeneration and tubular basement membrane segmental thickening.

Abbreviations: GBM, glomerular basement membrane; H\&E, hematoxylin \& eosin; PAS, periodic acid-Schiff; PASM, periodic acid-silver motheramine. 


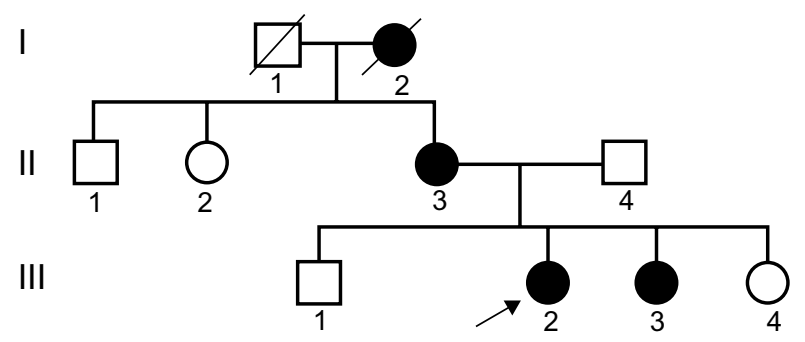

Figure 2 Pedigree chart of the $\mathrm{X}$-linked Alport syndrome. Notes: $\square$ normal male; $\bigcirc$ normal female; $\square$ male patient; $\bullet$ female pateient; $ð$ male death; $\sim$ - female' death; $\rightarrow$ proposita.

and stored it in a sterile beaker that contained $5 \mathrm{~mL}$ of penicillin-streptomycin antibiotics. The urine was stored at $4^{\circ} \mathrm{C}$ in a refrigerator and then subjected to the next step of the experiment within one day. The urine was dispensed into four centrifuge tubes that were centrifuged at $400 \times g$ for 100 minutes. There was $5 \mathrm{~mL}$ or less of urine in each tube after the supernatant was discarded. The remaining liquid was pooled into centrifuge tubes and $20 \mathrm{~mL}$ of phosphate-buffered saline (PBS) that contained $1 \%$ double antigens was then added. The mixed liquid was centrifuged at $400 \times g$ for 10 minutes, and after the supernatant was discarded, the remaining $1 \mathrm{~mL}$ or less of liquid contained the precipitate. The cell suspension was then transferred to 6 -well plates coated with $0.1 \%$ gelatin and incubated at $37^{\circ} \mathrm{C}$ under $5 \% \mathrm{CO}_{2}$ for 3 days. During the 3 days, the seeded cells were observed carefully; contaminated cells were discarded and $1 \mathrm{~mL}$ of LMM101 medium was added to the remaining cells. The medium was changed when it turned yellow. The supernatant was discarded and the plate was flushed with PBS three times. Further, $2 \mathrm{~mL}$ of medium was added to the plate and cell attachment was observed under light microscopy. When visible colonies appeared and internal cell fusion was completed, the urine cells were considered to be successfully cultured; they were then subjected to subculture. The details of the urine cell culture have been described previously. ${ }^{10}$

\section{iPSC generation}

We used a lentivirus that contained human complementary DNA (cDNA) encoding Oct4, Sox2, c-myc, and Klf4 to infect urine cells. Human cDNA encoding Oct4, Sox 2, c-myc, and Klf4 was cloned into the LeMIE vector, and the lentivirus was used to infect HEK2937T cells (Addgene). We also added polybrene to the medium to increase the infective efficiency. After 24 hours of infection, urine cells were seeded onto a layer of feeder cells. At day 2, the cells were fed with dialyzed fetal bovine serum (DFBS), and the medium culture supernatants were changed every day until day 6. On day 7 , the medium was changed to DFBS medium, which was changed daily. We used DFBS medium from day 7 to day 17 . At day 18, the medium was replaced with mTesR1 medium, which was renewed daily. At day 21, the colony morphology was identified to be hESC-like. We were able to pick up the colonies, and one clone was allocated to two 96-well plates. One plate was used for alkaline phosphatase (AP) staining of colonies that had a typical morphology, identical to that of hESCs, and the other plate was used to expand the cultures in hESC medium. The details of iPSC generation were described previously. ${ }^{10,11}$

\section{AP staining and immunocytochemistry}

Because both iPSCs and hESCs highly express AP, we used AP staining for the preliminary evaluation of iPSCs, which was performed using the AP detection kit (Millipore). Briefly, we used PBS to wash the colonies three times, after which they were fixed with 4\% PFA (phosphate buffered saline) for 2 minutes. The PFA was removed, and the colonies were washed two times with TBST buffer (20 mM Tris-HCl, $150 \mathrm{mM} \mathrm{NaCl}$, and $0.05 \%$ Tween 20 ). AP buffer was added to the colonies and incubated for 5 minutes. We prepared the AP substrate solution in the dark: $18 \mu \mathrm{L}$ of NBT (nitroblue tetrazolium) and $28 \mu \mathrm{L}$ of BCIP (5-Bromo-4-chloro-3-indolyl phosphate) were added

Table I The results of the blood examination, urine routine test, and blood biochemistry as well as the general information for the members of the Alport syndrome family

\begin{tabular}{|c|c|c|c|c|c|c|c|c|c|c|}
\hline Patient & Sex & Age & $\begin{array}{l}\text { Clinical } \\
\text { features }\end{array}$ & BLO & PRO & $\begin{array}{l}\text { Inosine } \\
(\mu \mathrm{mol} / \mathrm{L})\end{array}$ & $\begin{array}{l}\text { ALB } \\
\text { (g/L) }\end{array}$ & $\begin{array}{l}\text { GFR } \\
\text { (mL/min) }\end{array}$ & $\begin{array}{l}\text { Hearing } \\
\text { loss }\end{array}$ & $\begin{array}{l}\text { Vision } \\
\text { loss (L/R) }\end{array}$ \\
\hline III2 & Female & 26 & CKD3 & ++ & + & $12 \mid$ & 35 & 48.4 & + & $0.2 / 1.0$ \\
\hline III3 & Female & 23 & CKD2 & + & + & 110 & 45 & 64.4 & + & $0.4 / 1.0$ \\
\hline II3 & Female & 51 & CKD4 & ++ & ++ & 231 & 43 & 26.8 & + & $0.2 / 0.8$ \\
\hline III & Male & 54 & Normal & - & - & 46 & 47 & 183.3 & - & I.5/I.5 \\
\hline II2 & Female & 58 & Normal & - & + & 53 & 48 & 118.1 & - & $1.0 / 1.0$ \\
\hline$\| 4$ & Male & 53 & Normal & - & - & 55 & 52 & 159.7 & - & $1.0 / 1.0$ \\
\hline IIII & Male & 21 & Normal & - & - & 56 & 45 & 174.4 & - & $1.5 / 1.0$ \\
\hline III4 & Female & 17 & Normal & - & - & 50 & 43 & 199.8 & - & I.2/I.I \\
\hline
\end{tabular}

Abbreviations: GFR, glomerular filtration rate; ALB, serum albumin; BLO, urine erythrocyte; PRO, urine protein; L, left; R, right. 
A

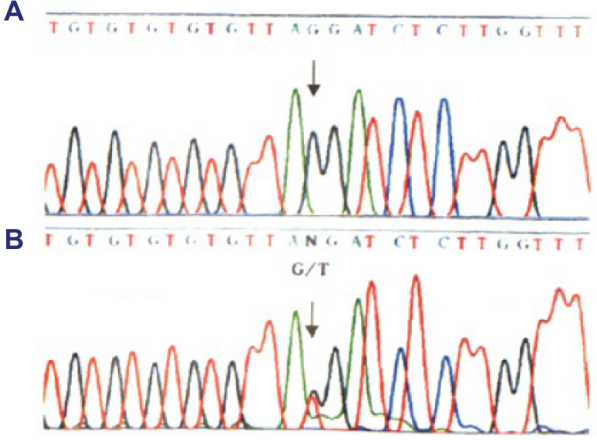

Figure 3 Identification of the c. |5|7-| G>T acceptor splicing site mutation on gene COL4A5.

Notes: (A) the sequence of healthy subject; (B) the mutation site, c. I5I7-I G>T in COL4A5 gene (arrow), base alteration from $G$ to $T$ on the acceptor splicing site of the 22 nd intron. Base $\mathrm{G}$ closed mutation was also found at the first base of the 23rd intron in COL4A5.

to $1 \mathrm{~mL}$ of AP buffer. Then, the colonies were stained with AP substrate solution in the dark for 15 minutes. After the AP staining, the colonies were observed under the microscope. We observed proteins that are highly expressed by iPSCs by using immunocytochemistry. The primary antibodies used in this study targeted Oct-4, SSAE-1, SSAE-4, TRA-1-60, and TRA-1-81. The cell nucleus was counterstained with DAPI (4',6-diamidino-2-phenylindole) (Sigma). The immunofluorescence outcomes were evaluated using fluorescent microscopy.

\section{Flow cytometry analysis}

We used flow cytometry to verify the pluripotency of the cells. Approximately $1.5 \times 10^{6}$ iPSCs were used for staining of the transcription factors (Oct-4, SSAE-4, TRA-1-60, and TRA1-81). After the disposure of the cell, we washed the cells with PBS and added $0.25 \%$ trypsin for several minutes to detach the cells. After centrifugation at $200 \times g$ for 5 minutes, we removed the supernatant and resuspended the cells with FACS (fluorescence activated cell sorter) buffer (PBS containing 2\% FBS). The cells were transferred to a $1.5 \mathrm{~mL}$ EP (Eppdndorf) tube and centrifuged at $200 \times g$ for 5 minutes. After centrifugation, the cells were washed with FACS two times and the supernatant was discarded. After washing, the cells were incubated with the primary antibody and the secondary antibody for 30 minutes at $37^{\circ} \mathrm{C}$. During the incubation, we agitated the EP tube to prevent the aggregation of cells on the bottom of tube, which could affect the antibody binding. After incubation and centrifugation at $200 \times g$ for 5 minutes, the cells were washed with FACS buffer. The supernatant was discarded and the cells were resuspended in FACS buffer. After filtration, flow cytometric analysis (Becton Dickinson FACScan, Mountain View, CA, USA) was performed.

\section{qRT-PCR analysis of genes iPSCs}

We used qRT-PCR to evaluate mRNA expression in cells. The gene expression profiles of iPSCs, hESCs, and urine cells were used to determine the identity of the iPSCs. Total RNA was extracted from urine cells, hESCs, and iPSCs according to the manufacturer's instructions (Thermo Fisher Scientific, Waltham, MA, USA). The RNA was reverse transcribed to cDNA with mRNA-specific stem-loop-like RT primers. The primers for the genes are listed in Table 2. Approximately $2.5 \mathrm{ng}$ of cDNA was then used for PCR on a Step One Plus machine. qRT-PCR was also used to confirm the specific endodermal, mesodermal, and ectodermal gene expression of hESCs. The primers for the genes representing the three embryonic germ layers are listed in Table 3.

\section{Karyotype analysis}

Karyotype analysis required approximately $3.5 \times 10^{6}$ cells with cell growth to $75 \%-85 \%$ density. The cells were incubated in a medium supplemented with $0.2 \mu \mathrm{g} / \mathrm{mL}$ colchicine under $5 \%$ $\mathrm{CO}_{2}$ at $37^{\circ} \mathrm{C}$ for 130 minutes. The cells were then washed with PBS two times. The cells were trypsinized to generate a single cell suspension. After centrifugation at $200 \times g$ for 5 minutes, the cells were treated with $7 \mathrm{~mL}$ of $\mathrm{KCl}$. The cells were then treated with $0.4 \%$ sodium citrate, $0.4 \%$ chloratum:kalium at a ratio of $1: 1$ for 18 minutes at $37^{\circ} \mathrm{C}$, and subsequently fixed in cool methanol:acetic acid (3:1) for 3 minutes. After fixation, the cells were resuspended in the fixative. The microslides were dried in a drying oven at $37^{\circ} \mathrm{C}$ for 3 hours. The chromosomes were stained with $5 \%$ Giemsa stain for 10 minutes and observed

Table 2 qRT-PCR primers used in the validation assays

\begin{tabular}{lll}
\hline $\begin{array}{l}\text { Primer } \\
\text { name }\end{array}$ & Sequence $\left(\mathbf{5}^{\prime} \mathbf{3}^{\prime}\right.$ ) & $\begin{array}{l}\text { Amplicon } \\
\text { length }\end{array}$ \\
\hline Oct-FI & TGTTCCTGCAGTGCCCGAAA & 109 \\
Oct-FI & TTCTGGCGCCGGTTACAGAA & \\
Sox2-FI & GAACCATCTCTGTGGTCTTG & 316 \\
Sox2-RI & GATTCTCGGCAGACTGATTC & \\
Kif4-FI & CCGCTCCATTACCAAGAGCT & 250 \\
Kif4-RI & TGGTCAGTTCATCTGAGCGG & \\
c-myc-FI & ACACATCAGCACAACTACGC & 159 \\
c-myc-RI & CCTCTTGACATTCTCCTCGGT & \\
Nanog-FI & TTCTGCTGAGATGCCTCACA & 322 \\
Nanog-RI & CATTCTCTGGTTCTGGAACC & \\
Lin28-FI & TGGGGTGTGTTTATTTGATGG & 96 \\
Lin28-RI & GGGAAGGGAAGAGTCAGCTT & \\
SALL4-FI & GACTCACACTGGAGAGAAGC & 170 \\
SALL4-RI & TTCCGTCCGTACCTAACAGA & \\
REXI-FI & CACAGTCCATCCTTACAGAGTT & 160 \\
REXI-RI & CGTTCAGTGCCTTCTCTATG & \\
GAPDH-FI & GGGAAACTGTGGCGTGAT & 299 \\
GAPDH-RI & GAGTGGGTGTCGCTGTTGA & \\
\hline
\end{tabular}


Table 3 qRT-PCR primers used in the validation assays for the three embryonic germ layers

\begin{tabular}{|c|c|c|}
\hline $\begin{array}{l}\text { Primer } \\
\text { name }\end{array}$ & Sequence $\left(5^{\prime}-3^{\prime}\right)$ & $\begin{array}{l}\text { Amplicon } \\
\text { length }\end{array}$ \\
\hline$\overline{A F P-F I}$ & AGCGGCTGACATTATTATCG & 150 \\
\hline AFP-RI & GCAGGAGGGACATATGTTTC & \\
\hline Amylase-FI & GGACCCAGTTTCCTTTCTTAGC & 169 \\
\hline Amylase-RI & CCAATGGTGAAAAGCAACCA & \\
\hline $\mathrm{NFH}-\mathrm{FI}$ & AGTCAAAGAGCCCCCAAA & 148 \\
\hline$N F H-R I$ & TTCGACAGCAGGTTCCTT & \\
\hline Enolase-FI & СССTTTGACCAGGATGACT & 130 \\
\hline Enolase-RI & TGCAGGACTTCTCGTTCAC & \\
\hline SoxI-FI & GGACTCTCTCTGAGGTTCTTTG & 130 \\
\hline SoxI-RI & GGCCCACATCCTAATCTTGA & \\
\hline c actin-FI & TGTGCTAGACAGGAACTCAGAT & 152 \\
\hline c actin-RI & GATGAGTCCAGAGTACTCCAAA & \\
\hline GAPDH-FI & GGGAAACTGTGGCGTGAT & 299 \\
\hline GAPDH-RI & GAGTGGGTGTCGCTGTTGA & \\
\hline
\end{tabular}

under a microscope. We first identified the split phase using the low-power lens. Subsequently, we observed the chromosomal alignment under the high-power immersion lens.

\section{Results}

\section{Generation of iPSCs}

We used lentiviral vectors that expressed the four pluripotency factors Oct4, Sox2, c-myc, and Klf4 to transduce renal tubular cells (Figure 4). At appropriately 7 days of culture, we observed early morphological changes indicative of reprogramming. The cell morphology generally changed from a long shuttle type to a large round type (Figure 4A-D). Small cell clusters appeared and became progressively larger each day. On day 14 , several colonies with $\mathrm{hESC}$ morphology were identified (Figure 4B). By day 21, compact colonies were formed (Figure 4C). Colonies were picked up and expanded for culture in MEF medium on day 20 as appropriate. At the end of this process, large and round colonies with defined boundaries were clearly observed (Figure 4D). The colonies stained positive for AP (Figure 4F). The iPSCs could be long-term subcultured in vitro; Figure $4 \mathrm{H}$ shows the colonies that were propagated to 12 generations. The colonies that were subcultured had the typical morphology of hESCs. Figure 4E represents primitive renal tubular cell. Figure 4G represents the iPSCs with AS staining.

\section{Expression of pluripotency markers in iPSCs}

Immunocytochemistry confirmed that iPSCs expressed several hESC-specific marker proteins (Oct4, SSAE-4, TRA-1-60, and TRA-1-81). However, the iPSCs were negative for SSAE-1 expression (Figure 5). qRT-PCR analyses of the total cellular RNA further confirmed the expression of pluripotencyassociated genes (Figure 6), including Oct4 (endogenous), Sox2 (endogenous), Klf4 (endogenous), c-myc (endogenous), Nanog, Lin28, SALL4, and REX1. Figure 6 shows that the pluripotency-related genes in iPSCs had a much higher expression than those in tubular cells and a similar expression to those in hESCs. These genes were expressed at almost undetectable levels in tubular cells. The exogenous pluripotency genes were evident in most of the clones. In contrast, the vector transgenes were silenced in derived iPSC clones. We also used flow cytometry to verify cell surface markers, including Oct4, SSEA-4, TRA-1-60, and TRA-1-81. iPSCs highly expressed cell surface markers associated with Oct4, SSEA-4, TRA-1-60, and TRA-1-81 (Figure 7). The expression of the pluripotency-associated genes indicated the successful generation of iPSCs from renal tubular cells.
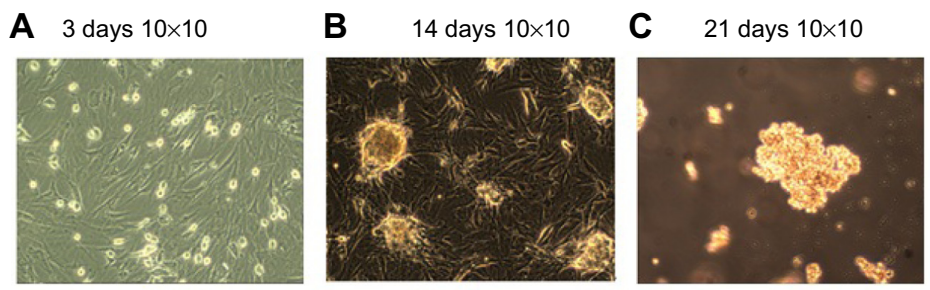

D After picking colonies $10 \times 10$
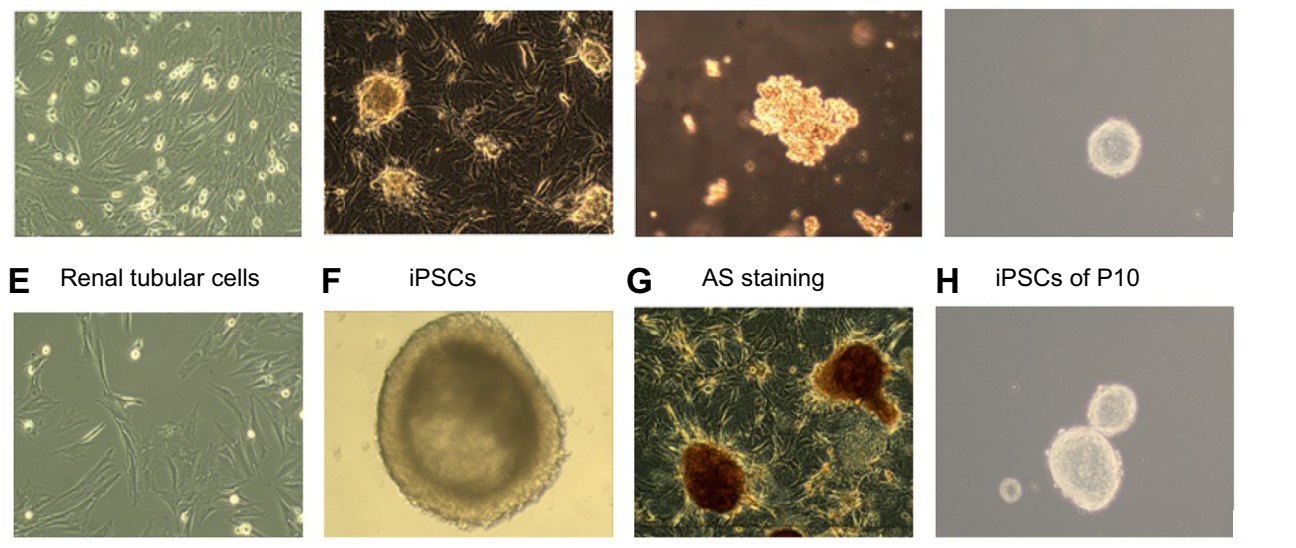

Figure 4 Schematic diagram of the renal tubular cell reprogramming procedure.

Notes: (A-D) Step-wise process of reprogramming renal tubular cells; (E) renal tubular cells; (F) iPSCs; (G) iPSCs with AS staining; and (H) iPSCs clones of ten generations. Abbreviations: AS, Alport syndrome; iPSCs, induced pluripotent stem cells. 

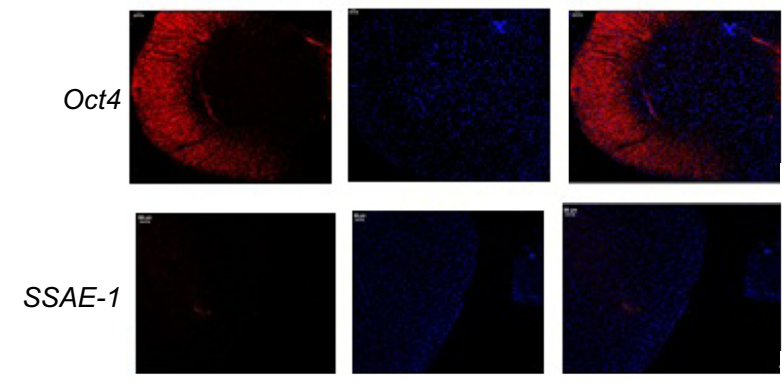

SSAE-4
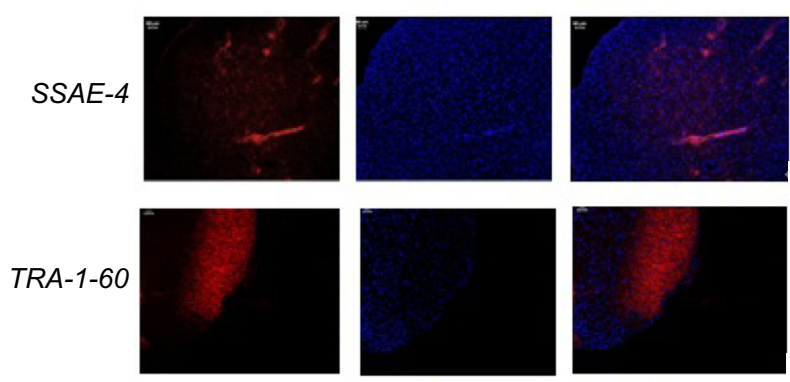

TRA-1-81
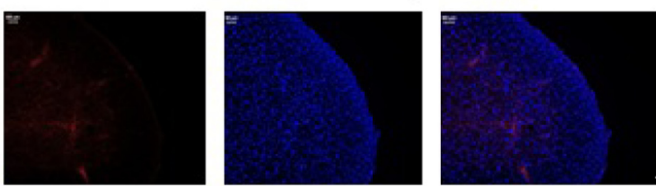

Figure 5 Immunohistochemical analysis of iPSCs.

Notes: In each specific marker proteins (Oct4, SSAE-I, SSAE-4, TRA-I-60, and TRA-I-8I), the pictures on the left represent the immunofluorescence test. The pictures in the middle represent the DAPI staining. The pictures on the right represent the overlapping images by left pictures and middle pictures.

Abbreviation: iPSCs, induced pluripotent stem cells.

\section{Pluripotency of reprogrammed cells and karyotype analysis}

iPSCs possessed the potential to differentiate into cystic embryoid bodies (EBs) in vitro. After adherent culture, the EBs began to spontaneously differentiate into cell types of the three embryonic germ layers, including endoderm, mesoderm, and ectoderm. The expression of genes (AFP, Amylase, c actin, Enolase, NFH, and Soxl) that were expressed by the three embryonic germ layers was verified by qRT-PCR. qRTPCR detected the specific gene expression of the EBs, endoderm, mesoderm, and ectoderm (Figure 8). In EBs formed at day 18 , the endoderm highly expressed the $A F P$ and amylase genes, the mesoderm highly expressed the $c$ actin and enolase genes, and the ectoderm highly expressed the $N F H$ and Sox 1 genes. Additionally, the karyotype of iPSCs was identical to that of renal tubular cells. Both these cell types represented the normal karyotype of a female: 46, XX (Figure 9).

\section{Discussion}

In this study, we used lentiviruses that expressed Oct4, Sox2, c-myc, and Klf4 to transduce renal tubular cells. After reprogramming, the cells presented the same

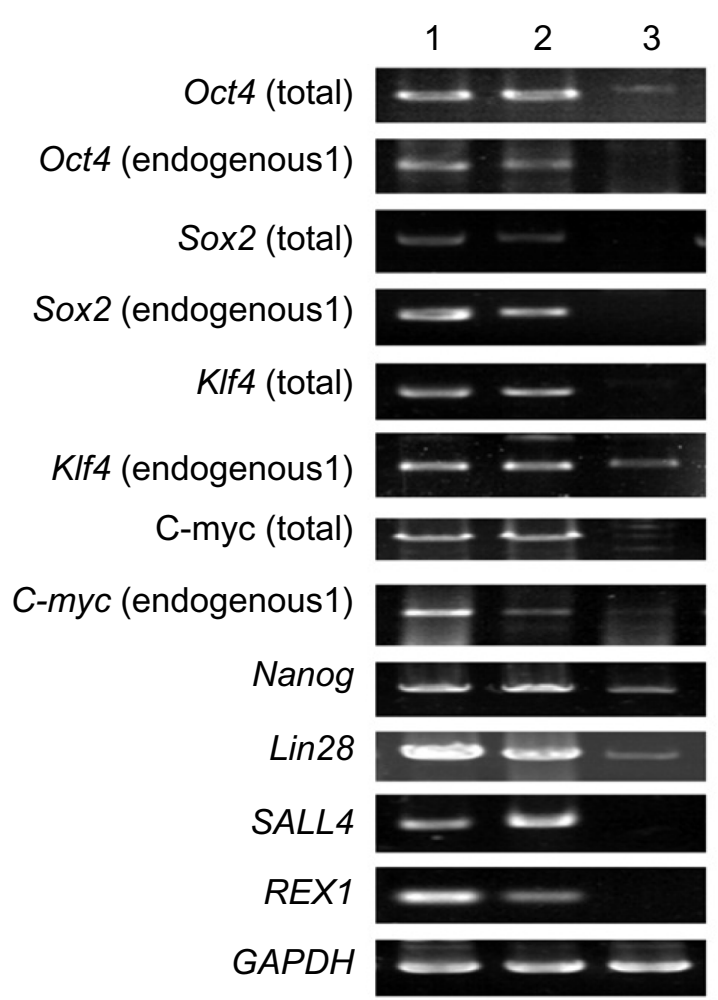

Figure 6 qRT-PCR analysis of gene expression in iPSCs.

Notes: I, iPSCs; 2, hESCs; 3, renal tubular cells.

Abbreviations: iPSC, induced pluripotent stem cells; hESC, human embryonic stem cell.
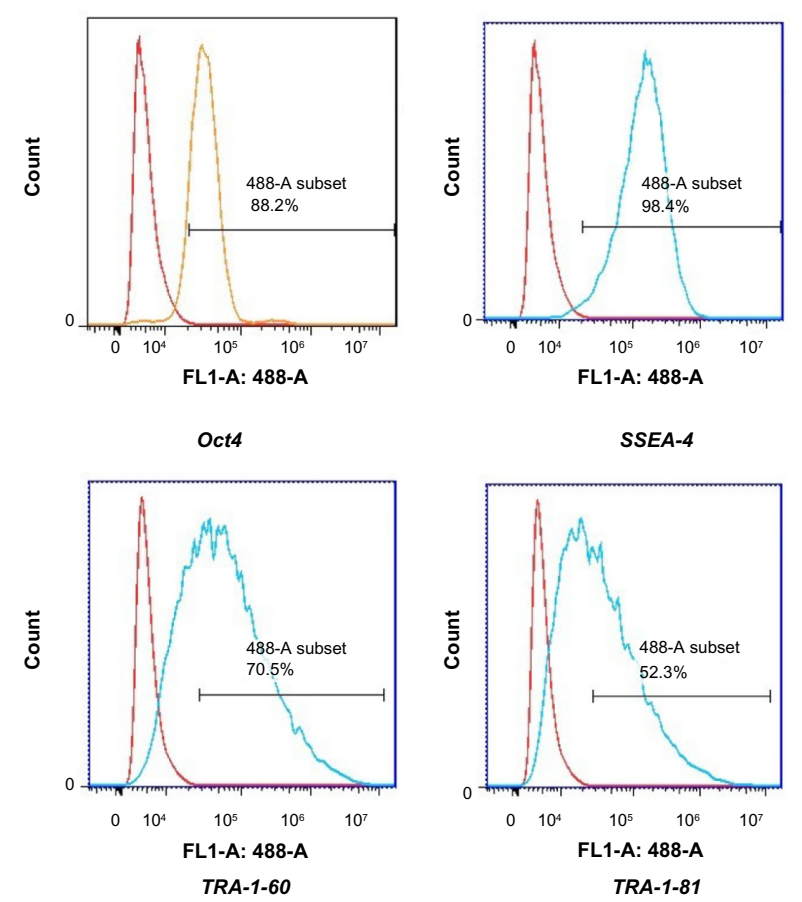

Figure 7 Flow cytometric analysis of the indicated markers in iPSCs.

Notes: The red histograms represented the negative peak curve and the blue histograms represented the positive peak curve. The horizontal line stood for the positive rate of detected cells.

Abbreviation: iPSCs, induced pluripotent stem cells. 


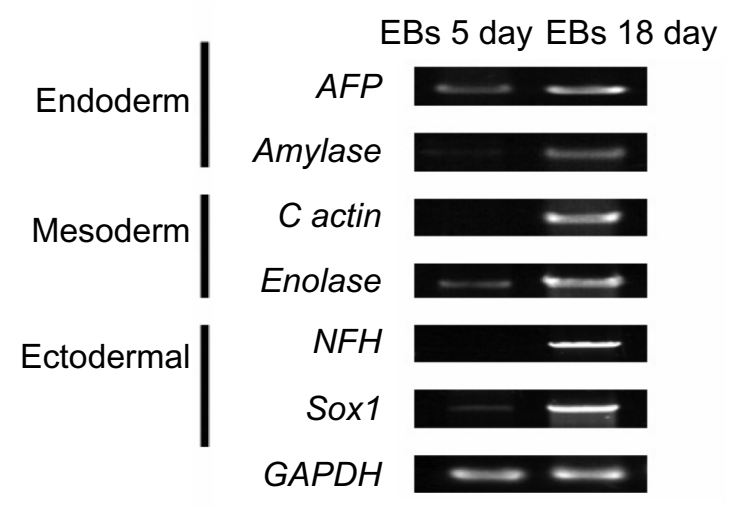

Figure 8 qRT-PCR detected the specific gene expression of endoderm, mesoderm, and ectoderm in EBs.

Abbreviation: EBs, embryoid bodies.

characteristics as hESCs with regard to morphological appearance, marker expression, and differentiation into the three embryonic germ layers. These results demonstrate that the renal tubular cells were reprogrammed and possessed the potential to generate iPSCs. The iPSCs generated from renal tubular cells were maintained for a long duration in vitro and maintained the biological characteristics of hESCs.

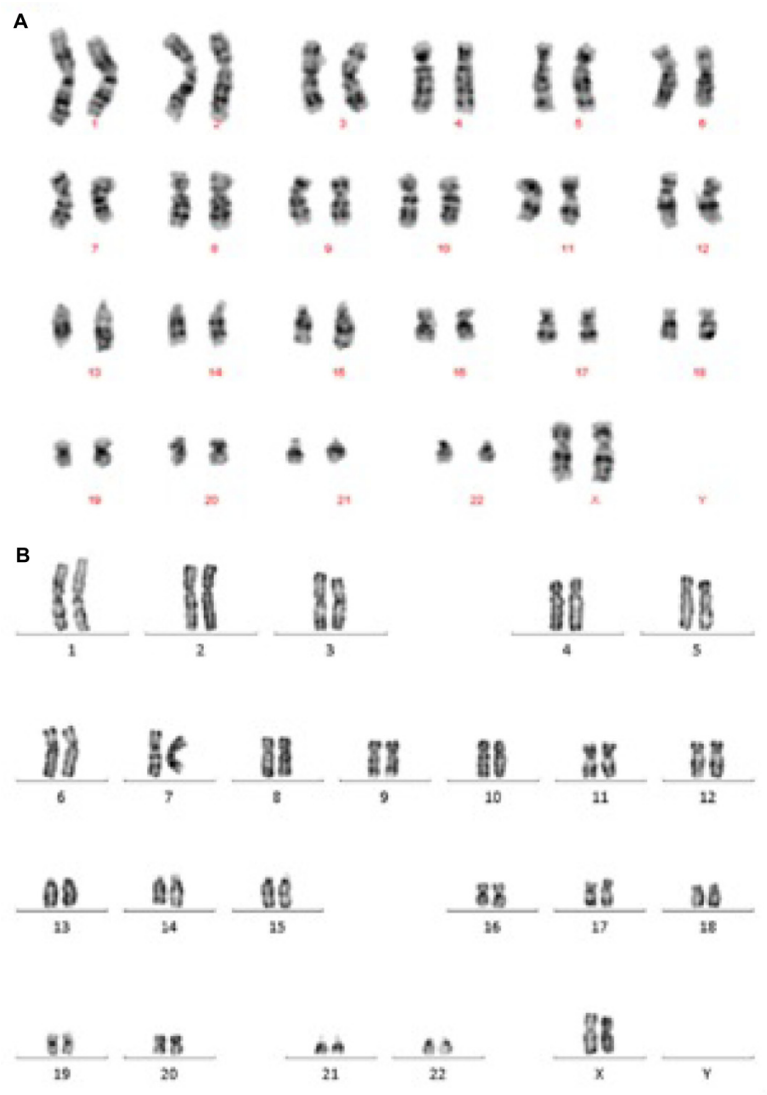

Figure 9 The karyotype of cells.

Notes: (A) Karyotype of renal tubular cells. (B) Karyotype of iPSCs derived from renal tubular cells.

Abbreviation: iPSCs, induced pluripotent stem cells.
All the experimental outcomes indicate that we successfully generated iPSCs from renal tubular cells. These iPSCs could provide a valuable resource for disease modeling and a good human genomic system for future studies investigating the pathogenesis of AS at the genetic level.

iPSCs have been a popular subject of global research since Takahashi et al first successfully generated iPSCs from human fibroblasts using defined factors. ${ }^{12}$ This approach not only overcame technological barriers, ethical issues, and traditional viewpoints, but also provided genetic information associated with disease pathogenesis, especially for diseases resulting from abnormal embryonic development. ${ }^{13,14}$ The conversion of human somatic cells derived from readily accessible tissue such as skin, blood, and nerve fibers into embryonic-like iPSCs has opened a new perspective for the modeling and understanding of many human pathologies. ${ }^{15}$ Currently, iPSC research is focused on numerous diseases, especially genetic diseases such as familiar Alzheimer's disease, Huntington's disease, and Parkinson's disease. ${ }^{16-18}$ In this study, we developed a simple, noninvasive, and acceptable method to generate iPSCs from renal tubular cells, thus providing an ideal cell source for iPSC generation. In contrast to other tissues sources such as skin and blood, which require a traumatic harvesting procedure, urine was easily accessible and was with minimum effort. We believe that renal tubular cells are ideal somatic cells for use in research in the future.

We generated iPSCs from the renal tubular cells of a patient with AS. We found that the culture conditions, choice of medium, and the exogenous induction material were important factors in the iPSC generation procedure. Any error in the procedure could lead to cell death or the failure of differentiation. iPSCs have been mostly generated by introducing Oct4, Sox2, c-myc, and Klf4. However, viral integration into the host genome increases the risk of tumorigenicity. Okita et al researched the generation of iPSCs without viral vectors. The production of virus-free iPSCs may provide a safe alternative in regenerative medicine research. ${ }^{19}$ Safe and effective direction of the fate of patient iPSCs has also been shown after administration of synthetic mRNAs. ${ }^{20}$ Vitamin C has the ability to improve iPSC generation and maintain hESCs in a blastocyst-like state because vitamin $\mathrm{C}$ regulates genes expressing numerous pluripotency factors, thereby increasing their promoter activity and protein levels. ${ }^{21,22}$ In our iPSCs, the induction of iPSCs was based on the introduction of the classical transcription factors Oct4, Sox2, c-myc, and Klf4. We could not confirm whether the generation of iPSCs using this technique would result in 
mutation or tumorigenicity, as the study duration was limited. Moreover, we found that iPSC generation was more effective when we added vitamin $\mathrm{C}$ to the medium.

Previously, urine cells and kidney mesangial cells were successfully reprogrammed..$^{23,24}$ Our group also generated iPSCs from the urine cells of a patient with systemic lupus erythematosus. ${ }^{7}$ The results obtained in the present study by using urine-derived AS iPSCs demonstrate that urine cells are among the most acceptable noninvasive sources of iPSCs, especially for research on genetic diseases.

Patients with AS inevitably present with kidney failure, for which the currently available treatment is either dialysis or kidney transplantation. However, the number of kidneys available for transplantation is limited and the cost of dialysis is high. The application of iPSCs to cell therapy may thus be useful to treat various diseases. iPSCs have the same capability as hESCs to regenerate tissues and even an entire organism. In conclusion, the present study established two novel points. First, we successfully generated iPSCs from the renal tubular cells of an AS patient, thereby laying a foundation to further research the pathogenesis of AS. Second, the iPSCs of the AS patient contained the appropriate genetic background, thus providing a unique platform for studies aimed at both epigenetic analysis and iPSC therapy.

\section{Acknowledgments}

The authors thank the members of the AS family who actively participated in our study. They also thank the South China Institute for Stem Cell Biology and Regenerative Medicine, the Guangzhou Institute of Biomedicine and Health, and the Chinese Academy of Sciences, Guangzhou, People's Republic of China, for their assistance in this study. The research was supported by Guangdong Shenzhen Knowledge Innovation Program basic Research items (JCYJ21040416122812045).

\section{Disclosure}

The authors report no conflicts of interest in this work.

\section{References}

1. Hertz JM. Alport syndrome. Molecular genetic aspects. Danish Med Bull. 2009;56(3):105-152.

2. Thorner PS. Alport syndrome and thin basement membrane nephropathy. Nephron Clin Pract. 2007;106(2):c82-c88.

3. Wang XP, Fogo AB, Colon S, et al. Distinct epitopes for anti-glomerular basement membrane alport alloantibodies and goodpasture autoantibodies within the noncollagenous domain of alpha3(IV) collagen: a janus-faced antigen. J Am Soc Nephrol. 2005;16(12):3563-3571.

4. Kruegel J, Rubel D, Gross O. Alport syndrome - insights from basic and clinical research. Nature Rev Nephrol. 2013;9(3):170-178.
5. Kashtan CE. Alport Syndrome and Thin Basement Membrane Nephropathy. In: Pagon RA, Adam MP, Ardinger HH, et al, editors. GeneReviews ${ }^{\circledR}$. Seattle, WA: University of Washington; 1993.

6. Haas M. Alport syndrome and thin glomerular basement membrane nephropathy: a practical approach to diagnosis. Arch Pathol Lab Med. 2009;133(2):224-232.

7. Chen Y, Luo R, XuY, et al. Generation of systemic lupus erythematosusspecific induced pluripotent stem cells from urine. Rheumatol Int. 2013;33(8):2127-2134.

8. Luo Y, Fan Y, Zhou B, Xu Z, Chen Y, Sun X. Generation of induced pluripotent stem cells from skin fibroblasts of a patient with olivopontocerebellar atrophy. Tohoku J Exp Med. 2012;226(2):151-159.

9. He XL, Dai Y, Wang SY, et al. X连锁显性遗传性肾炎一家系相关基 因排除定位分析 [A Exclusive Mapping Analysis on the Gene Associated with X-linked Dominant Inherited Nephritis in One Pedigree]. 中国医师杂志 [Journal of Chinese Physician]. 2004;6(11):1508-1510. Chinese.

10. Zhou T, Benda C, Duzinger S, et al. Generation of induced pluripotent stem cells from urine. J Am Soc Nephrol. 2011;22(7):1221-1228.

11. Xue Y, Cai X, Wang L, et al. Generating a non-integrating human induced pluripotent stem cell bank from urine-derived cells. PloS One. 2013;8(8):e70573.

12. Takahashi K, Tanabe K, Ohnuki M, et al. Induction of pluripotent stem cells from adult human fibroblasts by defined factors. Cell. 2007;131(5):861-872.

13. Bahadur G, Morrison M, Machin L. Beyond the 'embryo question': human embryonic stem cell ethics in the context of biomaterial donation in the UK. Reprod Biomed Online. 2010;21(7):868-874.

14. Gauthier M, Maury Y, Peschanski M, Martinat C. Human pluripotent stem cells for genetic disease modeling and drug screening. Regener Med. 2011;6(5):607-622.

15. Maury Y, Gauthier M, Peschanski M, Martinat C. Human pluripotent stem cells for disease modelling and drug screening. BioEssays: News Rev Mol Cell Dev Biol. 2012;34(1):61-71.

16. Sanders LH, Laganiere J, Cooper O, et al. LRRK2 mutations cause mitochondrial DNA damage in iPSC-derived neural cells from Parkinson's disease patients: reversal by gene correction. Neurobiol Dis. 2014;62:381-386.

17. Mohamet L, Miazga NJ, Ward CM. Familial Alzheimer's disease modelling using induced pluripotent stem cell technology. World J Stem Cells. 2014;6(2):239-247.

18. Fink KD, Crane AT, Leveque $X$, et al. Intrastriatal transplantation of adenovirus-generated induced pluripotent stem cells for treating neuropathological and functional deficits in a rodent model of Huntington's disease. Stem Cells Transl Med. 2014;3(5):620-631.

19. Okita K, Nakagawa M, Hyenjong H, Ichisaka T, Yamanaka S. Generation of mouse induced pluripotent stem cells without viral vectors. Science. 2008;322(5903):949-953.

20. Warren L, Manos PD, Ahfeldt T, et al. Highly efficient reprogramming to pluripotency and directed differentiation of human cells with synthetic modified mRNA. Cell Stem Cell. 2010;7(5):618-630.

21. Esteban MA, Wang T, Qin B, et al. Vitamin C enhances the generation of mouse and human induced pluripotent stem cells. Cell Stem Cell. 2010;6(1):71-79.

22. Gao Y, Yang L, Chen L, et al. Vitamin C facilitates pluripotent stem cell maintenance by promoting pluripotency gene transcription. Biochimie. 2013;95(11):2107-2113.

23. Song B, Niclis JC, Alikhan MA, et al. Generation of induced pluripotent stem cells from human kidney mesangial cells. J Am Soc Nephrol. 2011;22(7):1213-1220.

24. Montserrat N, Ramirez-Bajo MJ, Xia Y, et al. Generation of induced pluripotent stem cells from human renal proximal tubular cells with only two transcription factors, OCT4 and SOX2.J Biol Chem. 2012;287(29): 24131-24138 
International Journal of Nephrology and Renovascular Disease

Dovepress

\section{Publish your work in this journal}

The International Journal of Nephrology and Renovascular Disease is an international, peer-reviewed open-access journal focusing on the pathophysiology of the kidney and vascular supply. Epidemiology, screening, diagnosis, and treatment interventions are covered as well as basic science, biochemical and immunological studies. The journal welcomes

original research, clinical studies, reviews \& evaluations, expert opinion and commentary, case reports and extended reports. The manuscript management system is completely online and includes a very quick and fair peerreview system, which is all easy to use. Visit http://www.dovepress.com/ testimonials.php to read real quotes from published authors.

Submit your manuscript here: http://www.dovepress.com/international-journal-of-nephrology-and-renovascular-disease-journal 